\title{
Two Partial Orders for Standard Young Tableaux
}

\author{
Justyna Kosakowska* \\ Faculty of Mathematics and Computer Science \\ Nicolaus Copernicus University \\ ul. Chopina 12/18, 87-100 Toruń, Poland. \\ justus@mat.umk.pl
}

\author{
Markus Schmidmeier $^{\dagger}$ \\ Department of Mathematical Sciences \\ Florida Atlantic University \\ 777 Glades Road, Boca Raton \\ Florida 33431, U.S.A. \\ markus@math . fau .edu
}

\author{
Hugh Thomas ${ }^{\ddagger}$ \\ LaCIM \\ Département de mathématiques \\ UQAM \\ C.P 8888, succursalle Centre-ville \\ PK-5151 \\ Montréal, QC H3C 3P8, Canada. \\ hugh.ross.thomas@gmail.com
}

Submitted: Jan 25, 2016; Accepted: May 26, 2019; Published: Jul 19, 2019

(C) The authors. Released under the CC BY-ND license (International 4.0).

\begin{abstract}
In this manuscript we show that two partial orders defined on the set of standard Young tableaux of shape $\alpha$ are equivalent. In fact, we give two proofs for the equivalence of the box order and the dominance order for tableaux. Both are algorithmic. The first of these proofs emphasizes links to the Bruhat order for the symmetric group and the second provides a more straightforward construction of the cover relations. This work is motivated by the known result that the equivalence of the two combinatorial orders leads to a description of the geometry of the representation space of invariant subspaces of nilpotent linear operators.
\end{abstract}

Mathematics Subject Classifications: 05E10, 47A15

*The first named author is partially supported by Research Grant No. DEC-2011/ 02/A/ ST1/00216 of the Polish National Science Center.

${ }^{\dagger}$ This research is partially supported by a Travel and Collaboration Grant from the Simons Foundation (Grant number 245848 to the second named author)

¥The third named author was partially supported by an NSERC Discovery Grant and the Canada Research Chairs program. 


\section{Introduction}

Let $\alpha$ be a partition. By $\mathcal{T}_{\alpha}$ we denote the set of all standard Young tableaux (SYT) of shape $\alpha$. In Section 2 we define two partial orders $\leqslant_{\text {box }}$ and $\leqslant_{\text {dom }}$ on the set $\mathcal{T}_{\alpha}$. These orders are defined combinatorially and are of importance in the theory of invariant subspaces of nilpotent linear operators. They control the geometry of varieties of invariant subspaces of nilpotent linear operators, as they describe the degeneration relation and the boundaries of the irreducible components, see $[4,5,6]$ and Section 3 . Therefore, it is important to investigate properties of these orders. One of the main results of the paper is the following theorem.

Theorem 1.1. Let $X, Z$ be SYTs of the same shape. The following conditions are equivalent.

1. $Z \leqslant$ box $X$,

2. $Z \leqslant \operatorname{dom} X$.

We show in [6] that several other relations of geometric or of algebraic nature lie between the box and the dominance relations. If those two are equal, then all the relations coincide.

We remark that partial orders on standard Young tableaux have been considered elsewhere, such as, notably, by Taşkin in [9]. Note, though, that the posets defined there are defined on all SYTs of size $n$ at once. In fact, for three of the partial orders considered there (the weak, KL, and geometric orders), two non-equal fillings of the same shape are always incomparable [9, Proposition 3.5], so the restriction to SYT of fixed shape is not interesting. The order which Taşkin refers to as chain order is similar to, but strictly weaker than, our dominance order (after transposition).

We present two different proofs of Theorem 1.1. Both proofs are constructive. The first one, presented in Section 4, shows connections of our problem with the Bruhat order in the symmetric group $S_{n}$ where $n=|\alpha|$.

The second proof, given in Section 5, gives a more straightforward algorithm that applies operations on entries of SYTs. Given two SYTs such that $Z \leqslant$ dom $X$, both algorithms compute a sequence of box moves that convert $X$ to $Z$. This proves that $Z \leqslant$ box $X$.

Finally, in Section 6 , we describe some properties of the poset $\left(\mathcal{T}_{\alpha}, \leqslant_{\text {box }}\right)$. We prove that there exists exactly one minimal and exactly one maximal element, and that all saturated chains have the same length.

\section{Definitions and notation}

Following $[2,7]$ we recall definitions and notation connected with SYTs. 
Notation: Recall that a partition $\alpha=\left(\alpha_{1}, \ldots, \alpha_{s}\right)$ is a finite non-increasing sequence of natural numbers; we picture $\alpha$ by its Young diagram which consists of $s$ rows of lengths given by the parts of $\alpha$. Let $|\alpha|=\alpha_{1}+\ldots+\alpha_{s}$. The transpose $\alpha^{\prime}$ of $\alpha$ is given by the formula

$$
\alpha_{j}^{\prime}=\#\left\{i: \alpha_{i} \geqslant j\right\},
$$

it is pictured by the transpose of the Young diagram for $\alpha$. Two partitions $\alpha, \widetilde{\alpha}$ are in the dominance partial order, in symbols $\alpha \leqslant_{\text {dom }} \widetilde{\alpha}$, if the inequality

$$
\alpha_{1}+\cdots+\alpha_{j} \leqslant \widetilde{\alpha}_{1}+\cdots+\widetilde{\alpha}_{j}
$$

holds for each $j$.

Notation: Let $\alpha$ be a partition. A standard Young tableau (SYT) of shape $\alpha$ is a tableau of shape $\alpha$ filled by the numbers $1,2, \ldots,|\alpha|$ such that its entries increase along rows and down columns. Let $\mathcal{T}_{\alpha}$ be the set of all SYTs of shape $\alpha$.

Given a SYT $X$ we number its rows starting from the top and going down.

Example: Let $\alpha=(2,2,1)$. There are exactly five SYTs of shape $\alpha$ :

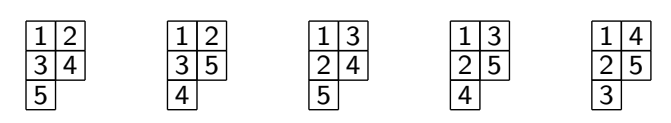

In the first tableau the entries 1 and 2 are in the row number 1 ; the entries 3,4 in the row number 2 , etc.

Notation: One can represent a SYT $X$ by a sequence of partitions

$$
X=\left[\gamma^{(1)}, \ldots, \gamma^{(|\alpha|)}\right]
$$

where $\gamma^{(i)}$ denotes the region in the Young diagram $\alpha$ which contains the entries $1, \ldots$, i .

In the example above, the first filling is given by the sequence of partitions $X=[(1),(2),(2,1),(2,2),(2,2,1)]$.

We introduce two partial orders on the set $\mathcal{T}_{\alpha}$ of all SYTs of the same shape.

Definition: Two SYTs $Z=\left[\delta^{(1)}, \ldots, \delta^{(|\alpha|)}\right], X=\left[\gamma^{(1)}, \ldots, \gamma^{(|\alpha|)}\right]$ of the same shape are $r e$ lated in the dominance partial order, in symbols $Z \leqslant_{\operatorname{dom}} X$, if for each $i$, the corresponding partitions $\delta^{(i)}, \gamma^{(i)}$ are related in the dominance partial order, i.e. $\delta^{(i)} \leqslant$ dom $\gamma^{(i)}$.

In the example above of SYTs of shape $(2,2,1)$, the first one listed is the largest in dominance order, and the last one is the smallest, while, for example, the second and third are incomparable in dominance order.

Definition: Suppose $X, Z$ are SYTs of the same shape. We say $Z$ is obtained from $X$ by a decreasing box move if $Z$ is obtained by swapping two entries in $X$ so that the smaller entry winds up in the higher-numbered row. We denote by $\leqslant_{\text {box }}$ the partial order generated by box moves. 
Here is an example:

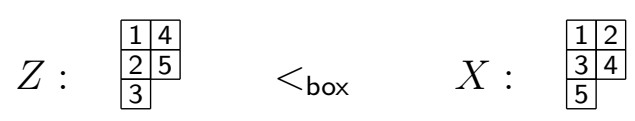

To get $Z$ we apply to $X$ the following sequence of moves. First we exchange 2 and 3 , then 4 and 5 and finally 3 and 4.

We finish this section by establishing the following fact.

Lemma 2.1. For SYTs of the same shape, the $\leqslant_{\text {box }}$-order implies the $\leqslant$ dom- order.

Proof. Suppose that the SYT $Z=\left[\delta^{(1)}, \ldots, \delta^{(|\alpha|)}\right]$ is obtained from $X=\left[\gamma^{(1)}, \ldots, \gamma^{(|\alpha|)}\right]$ by a decreasing box move based on swapping entries $a$ and $b$ with, say, $a<b$. Note that the partitions $\gamma^{(1)}, \ldots, \gamma^{(a-1)}$, and $\gamma^{(b)}, \ldots, \gamma^{(|\alpha|)}$ remain unchanged. The partitions $\delta^{(\ell)}$, $\gamma^{(\ell)}$ for $a \leqslant \ell<b$ are different and satisfy $\delta^{(\ell)}<_{\text {dom }} \gamma^{(\ell)}$ (since the defining partial sums can only decrease). This shows that $Z<_{\text {dom }} X$.

\section{LR-fillings and motivation}

Our investigation of partial orders for SYTs is motivated by its connections with LRfillings and an application to short exact sequences of nilpotent linear operators.

Fix two partitions $\gamma \subseteq \beta$ such that the Young diagram for $\gamma$ is contained in the Young diagram for $\beta$. The skew diagram $\beta \backslash \gamma$ is said to be a vertical strip if $\beta_{i} \leqslant \gamma_{i}+1$ holds for all $i$, and a horizontal strip if $\beta^{\prime} \backslash \gamma^{\prime}$ is a vertical strip. A rook strip is a horizontal and vertical strip.

Given three partitions $\alpha=\left(\alpha_{1}, \ldots, \alpha_{s}\right), \beta, \gamma$, we will consider fillings of $\beta \backslash \gamma$ which have $\alpha_{1}$ entries 1's, $\alpha_{2}$ entries 2's, etc. We describe such a filling as having the content $\alpha$ and the shape $\beta \backslash \gamma$. The type of the filling, $(\alpha, \beta, \gamma)$, records the content and shape. A filling is said to be an $L R$-filling if the following three conditions are satisfied:

- in each row, the entries are weakly increasing,

- in each column, the entries are strictly increasing,

- (lattice permutation property) for each $u>1$ and for each column $c$ : on the right hand side of $c$, the number of entries $u-1$ is at least the number of entries $u$.

The number of LR-fillings of type $(\alpha, \beta, \gamma)$ is the Littlewood-Richardson coefficient $c_{\alpha, \gamma}^{\beta}$ which plays an important rôle in symmetric functions, Schubert calculus, and the representation theory of the general linear group, see $[2,7]$.

Example: Let $\alpha=(2,2,1), \beta=(4,3,3,2,1), \gamma=(3,2,2,1)$. We have to fill the skew diagram $\beta \backslash \gamma$ with two 1 's, two 2 's, and one 3 . Due to the conditions on an LR-filling, this can be done in exactly three ways.
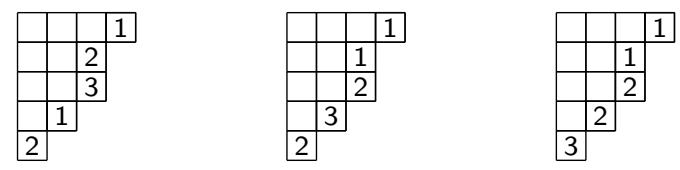
In this example, $\beta \backslash \gamma$ is a vertical but not a horizontal strip.

Let $\beta \backslash \gamma$ be a rook strip. Note that there is a bijection between the set $\mathcal{T}_{\alpha, \gamma}^{\beta}$ of LRfillings of type $(\alpha, \beta, \gamma)$ and the set $\mathcal{T}_{\alpha}$ of the SYTs of shape $\alpha$. A bijection $\Phi_{\gamma}^{\beta}: \mathcal{T}_{\alpha, \gamma}^{\beta} \rightarrow \mathcal{T}_{\alpha}$ is constructed as follows. For an LR-filling $X$ we denote by $\tau(X)=\left(\tau_{1}, \ldots, \tau_{|\alpha|}\right)$ the list of entries when reading columns from the top down, starting with the rightmost column and moving left. Fix $i \leqslant s$, where $s$ is the number of rows of $\alpha$. Let $j_{1}<j_{2}<\ldots<j_{n_{i}}$ be all elements $j$ such that $\tau_{j}=i$. We write the elements $j_{1}, j_{2}, \ldots, j_{n_{i}}$ in the $i$-th row of the corresponding SYT of shape $\alpha$.

Box order and dominance order were defined on LR-fillings in [6]. We will review these definitions, and verify that they agree under the bijection defined above with the orders we have already defined on standard tableaux.

The box order is defined on LR-fillings of type $(\alpha, \beta, \gamma)$ by saying that the filling $Z$ is obtained by a decreasing box move from the filling $X$ if $Z$ is obtained from $X$ by swapping two entries, such that the smaller entry winds up in the lower position.

Lemma 3.1. For LR-fillings $X, Z$ of type $(\alpha, \beta, \gamma)$ we have that $\Phi_{\gamma}^{\beta}(X) \geqslant_{\text {box }} \Phi_{\gamma}^{\beta}(Z)$ if and only if $X \geqslant_{\text {box }} Z$.

Proof. Let $X, Z$ be LR-fillings of type $(\alpha, \beta, \gamma)$. Suppose we obtain $Z$ from $X$ by swapping the positions of entries $\tau_{i}$ and $\tau_{j}$ with $i<j$, and in $X$ we have $\tau_{i}=a, \tau_{j}=b$, with $a<b$.

Now consider $\Phi_{\gamma}^{\beta}(X)$ and $\Phi_{\gamma}^{\beta}(Z)$. In $\Phi_{\gamma}^{\beta}(X)$, we have $i$ in row $a$ and $j$ in row $b$. By the definition of $\Phi_{\gamma}^{\beta}$, we see that we obtain $\Phi_{\gamma}^{\beta}(Z)$ from $\Phi_{\gamma}^{\beta}(X)$ by swapping the entries $i$ and $j$ and resorting the two rows if necessary so that they are increasing. This resorting step is not allowed in our definition of decreasing box moves for SYTs.

However, it turns out that there is a sequence of legal decreasing box moves which suffice to transform $\Phi_{\gamma}^{\beta}(X)$ into $\Phi_{\gamma}^{\beta}(Z)$.

Let $I$ be the entries of row $a$ of $\Phi_{\gamma}^{\beta}(X)$ which are weakly between $i$ and $j$, and similarly let $J$ be the entries of row $b$ of $\Phi_{\gamma}^{\beta}(X)$ which are weakly between $i$ and $j$. The proof is by induction on $|I|$.

The base case is when $|I|=1$, in which case $I=\{i\}$. In this case, if $J=\left\{j_{r}<j_{r-1}<\right.$ $\cdots<j_{1}=j$, perform the following sequence of swaps:

$$
\left(i, j_{r}\right),\left(j_{r}, j_{r-1}\right), \ldots\left(j_{2}, j_{1}\right)
$$

This has the effect of swapping $i$ and $j$ and resorting row $b$.

Now suppose that $|I|=p>1$. Let the largest element of $I$ be $i_{p}$, and let $J^{\prime}=\left\{j_{r}<\right.$ $\left.\cdots<j_{2}<j_{1}=j\right\}$ be the elements of row $b$ which are weakly between $i_{p}$ and $j$. Perform the same sequence of swaps as before using $i_{p}$ and $J^{\prime}$ in place of $i$ and $J$. This has the effect of swapping $i_{p}$ and $j$, and resorting row $b$ if necessary. To complete the desired effect, it suffices to swap $i$ and $i_{p}$ (which is now in row $b$ ). This is another instance of the same problem, but with the original $i_{p}$ having been removed from $I$. We are therefore done by induction.

The fact that all the tableaux we pass through are indeed standard, follows from the fact that the starting and ending tableaux are standard by hypothesis. 
The converse direction is obvious: if there is a decreasing box move from $\Phi_{\gamma}^{\beta}(X)$ to $\Phi_{\gamma}^{\beta}(Z)$, then the corresponding move from $X$ to $Z$ is a decreasing box move.

For an example of the phenomenon considered in the previous proof, consider the following LR-fillings, which are related by the box move swapping the entries in rows 3 and 6 .

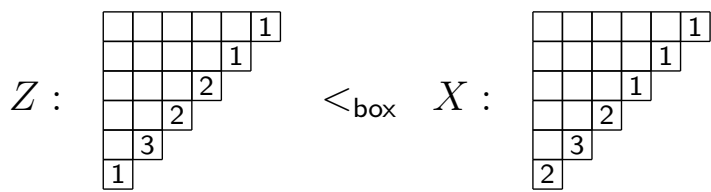

The corresponding tableaux are

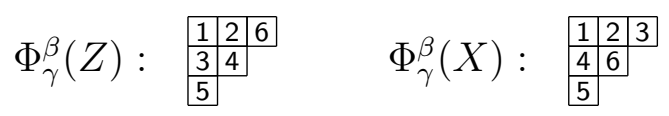

Swapping 3 and 6 is not a legal decreasing box move starting from $\Phi_{\gamma}^{\beta}(X)$, but we can first swap 3 and 4 , then 4 and 6 .

Similarly, one can define dominance order on the set of LR-fillings. One can represent an LR-filling $X$ by a sequence of partitions

$$
X=\left[\gamma^{(0)}, \ldots, \gamma^{(s)}\right]
$$

where $s$ is the number of rows of $\alpha$ and $\gamma^{(i)}$ denotes the region in the Young diagram $\beta$ which contains the entries $\square, 1, \ldots, i$. If $X$ has type $(\alpha, \beta, \gamma)$, then $\gamma=\gamma^{(0)}, \beta=\gamma^{(s)}$, and $\alpha_{i}=\left|\gamma^{(i)} \backslash \gamma^{(i-1)}\right|$ for $i=1, \ldots, s$. For LR-fillings, we define $X \geqslant$ dom $Z$ to mean that for all $1 \leqslant i \leqslant s$, the $i$-th partition corresponding to $X$ is greater than or equal to the $i$-th partition corresponding to $Z$ in dominance order.

We then have the following lemma:

Lemma 3.2. For LR-fillings $X, Z$ of type $(\alpha, \beta, \gamma)$ we have $\Phi_{\gamma}^{\beta}(X) \geqslant_{\mathrm{dom}} \Phi_{\gamma}^{\beta}(Z)$ if and only if $X \geqslant \mathrm{dom} Z$.

Proof. Suppose that $\alpha$ has $s$ rows. For $1 \leqslant r \leqslant s$, write $\left.\Phi_{\gamma}^{\beta}(X)\right|_{\leqslant r}$ for the first $r$ rows of $\Phi_{\gamma}^{\beta}(X)$. For $x$ a word, write sort $(x)$ for the result of sorting the letters of $x$ into increasing order. For a tableau $T$, sort $(T)$ denotes the list of entries of $T$ sorted into increasing order.

$X \geqslant{ }_{\text {dom }} Z$ is equivalent to saying that, for each $1 \leqslant r \leqslant s$, we have $\operatorname{sort}\left(\left.\Phi_{\gamma}^{\beta}(X)\right|_{\leqslant r}\right) \leqslant$ $\operatorname{sort}\left(\left.\Phi_{\gamma}^{\beta}(Z)\right|_{\leqslant r}\right)$, where the comparison is done coordinatewise.

Let $\delta^{(i)}$ be the sequence of partitions corresponding to $\Phi_{\gamma}^{\beta}(X)$, and let $\eta^{(i)}$ be the sequence of partitions corresponding to $\Phi_{\gamma}^{\beta}(Z)$.

The condition that, for a fixed $r$, we have $\operatorname{sort}\left(\left.\Phi_{\gamma}^{\beta}(X)\right|_{\leqslant r}\right) \leqslant \operatorname{sort}\left(\left.\Phi_{\gamma}^{\beta}(Z)\right|_{\leqslant r}\right)$, is equivalent to saying that, for all $i$, the sum of the first $r$ parts of $\delta^{(i)}$ is greater than or equal to the sum of the first $r$ parts of $\eta^{(i)}$. This condition for all $r$ is exactly the definition of $\Phi_{\gamma}^{\beta}(X) \geqslant_{\text {dom }} \Phi_{\gamma}^{\beta}(Z)$.

Let $k$ be an algebraically closed field. A nilpotent $k$-linear operator $N=(V, T)$ consists of a finite dimensional $k$-vector space $V$ together with a nilpotent $k$-linear map 
$T: V \rightarrow V$. Such an operator $N_{\alpha}=(V, T)$ is given uniquely, up to isomorphy, by a partition $\alpha$ recording the sizes of the Jordan blocks of the action of $T$ on the vector space $V$. Given two nilpotent linear operators $N=(V, T)$ and $N^{\prime}=\left(V^{\prime}, T^{\prime}\right)$, a morphism from $N$ to $N^{\prime}$ is a $k$-linear map $\phi: V \rightarrow V^{\prime}$ such that $T^{\prime} \phi=\phi T$.

The Green-Klein Theorem [3] establishes the link with LR-fillings:

Theorem 3.3. For partitions $\alpha, \beta, \gamma$, there exists a short exact sequence $0 \rightarrow N_{\alpha} \rightarrow$ $N_{\beta} \rightarrow N_{\gamma} \rightarrow 0$ of nilpotent linear operators if and only if there is an LR-filling of type $\left(\alpha^{\prime}, \beta^{\prime}, \gamma^{\prime}\right)$.

More precisely, if $A$ is the image of the embedding $N_{\alpha} \rightarrow N_{\beta}=B$ in the short exact sequence, then the tableau $X=\left[\gamma^{(0)}, \ldots, \gamma^{(s)}\right]$ of the sequence is given by $s=\min \{\ell$ : $\left.T^{\ell} A=0\right\}$, and the transposes of the partitions $\gamma^{(\ell)}$ are given by the Jordan types of the action of $T$ on the factors $B / T^{\ell} A \cong N_{\left(\gamma^{(\ell)}\right)^{\prime}}$.

Together, the $k[T]$-monomorphisms in the short exact sequences form a constructible subset $\mathbb{V}_{\alpha, \gamma}^{\beta}$ of the affine variety $\operatorname{Hom}_{k}\left(N_{\alpha}, N_{\beta}\right)$. Note that each irreducible component $\overline{\mathbb{V}}_{X}$ of $\mathbb{V}_{\alpha, \gamma}^{\beta}$ is given as the closure of the set of sequences with corresponding LR-filling $X$. All irreducible components have the same dimension.

Definition: Two LR-tableaux $X, Z$ of the same type are said to be in boundary relation, $Z \preccurlyeq$ boundary $X$, if $\mathbb{V}_{X} \cap \overline{\mathbb{V}}_{Z} \neq \varnothing$ holds.

The following theorem is shown in [6]:

Theorem 3.4. Suppose $X, Y$ are LR-tableaux of the same type and of shape which is a rook strip. If $Y$ is obtained from $X$ by a decreasing box move, then $Y \prec_{\text {boundary }} X$.

More precisely, given $X, Y$ in box relation, we construct a one-parameter family of embeddings $M(\lambda)$, and for each embedding a short exact sequence $0 \rightarrow L \rightarrow M(\lambda) \rightarrow$ $N \rightarrow 0$, such that the following properties are satisfied:

1. $L \oplus N$ has tableau $X$;

2. the sequence is split exact if $\lambda=0$;

3. $M(\lambda)$ has tableau $Y$ if $\lambda \neq 0$.

Thus, the above result provides a link between the combinatorial relation given by box moves, the algebraic relation given by short exact sequences and the geometric boundary relation.

In general, the boundary relation implies the dominance relation [6]. Hence, the transitive closure $\leqslant_{\text {boundary }}$ of the boundary relation $\preccurlyeq_{\text {boundary }}$ is a partial order.

We obtain from Theorem 3.4 the following chain of implications for tableaux $X, Z$ of the same type such that the box relation is defined:

$$
Z \leqslant_{\text {box }} X \text { implies } Z \leqslant_{\text {boundary }} X \text { implies } Z \leqslant \text { dom } X
$$

As a consequence, Theorem 1.1 yields the following result: 
Theorem 3.5. The following statements are equivalent for LR-tableaux $X, Z$ of the same type and of shape which is a rook strip.

$$
\begin{aligned}
& \text { 1. } Z \leqslant \text { box } X \\
& \text { 2. } Z \leqslant \text { boundary } X \\
& \text { 3. } Z \leqslant \text { dom } X
\end{aligned}
$$

The case where the partition $\alpha$ has at most two parts is particularly well understood since then for each shape $\beta \backslash \gamma$, there are only finitely many isomorphism classes of short exact sequences in $\mathbb{V}_{\alpha, \gamma}^{\beta}$. In this situation, the boundary relation has a combinatorial description in terms of arc diagrams, see [4, Theorem 1.2], and is, in fact, transitive and equivalent to several algebraic and combinatorial relations, in particular to $\leqslant_{\text {box }}$ and $\leqslant$ dom.

\section{The Bruhat order and the first proof of the main result}

Notation: Let $S_{n}$ denote the symmetric group on $n$ letters. For any $i=1, \ldots, n-1$, denote by $s_{i}=(i, i+1) \in S_{n}$ the adjacent transposition. Let $x \in S_{n}$ and let $x=s_{i_{1}} s_{i_{2}} \cdots s_{i_{k}}$ with $k$ minimal. Such an expression for $x$ is called reduced. The number $k$ is called the length of $x$ and we denote it by $\ell(x)=k$.

A key ingredient for our first proof of the main result is the notion of Bruhat order on the symmetric group. An excellent reference for this topic is [1]. There are several ways to define Bruhat order on $S_{n}$. We define the Bruhat graph on $S_{n}$ by putting an edge from $u$ to $v$ if $\ell(u)<\ell(v)$ and $u t=v$ for $t$ a transposition; we then define $u \leqslant v$ if there is a path from $u$ to $v$ in the Bruhat graph. It follows directly from this definition that $u<v$ if and only if $u^{-1}<v^{-1}$, using the fact that if $v=u t$, then $v^{-1}=u^{-1}\left(u t u^{-1}\right)$, where $u t u^{-1}$ is also a transposition. We call $v$ a cover of $u$ (and write $u \lessdot v$ ) if $u<v$ and there is no $w$ such that $u<w<v$.

The following well-known lemma characterizes the cover relations in Bruhat order.

Lemma 4.1 ([1, Lemma 2.1.4, Theorem 2.2.6]). For $u, v$ in $S_{n}$, the following conditions are equivalent:

- $u \lessdot v$,

- $\ell(v)=\ell(u)+1$ and $v=u t$ for $t$ some transposition,

- $v=u(a, b)$, where $u(a)<u(b)$ and for $a<i<b$, we do not have $u(a)<u(i)<u(b)$.

We will also need two other characterizations of Bruhat order.

Theorem 4.2 ([1, Theorem 2.2.2]). For $u, v$ in $S_{n}$, let $s_{i_{1}} \ldots s_{i_{p}}$ be a reduced expression for $v$. Then $u \leqslant v$ if and only if there is a subword of $s_{i_{1}} \ldots s_{i_{p}}$ which is a reduced expression for $u$. 
For a sequence of integers $\left(a_{1}, \ldots, a_{r}\right)$, we write $\operatorname{sort}\left(a_{1}, \ldots, a_{r}\right)$ for the sequence sorted into increasing order.

Theorem 4.3 ([1, Theorem 2.6.3]). For $u, v \in S_{n}$, we have $u<v$ if and only if for all $1 \leqslant i \leqslant n$, we have $\operatorname{sort}(u(1), \ldots, u(i))$ has each entry less than or equal to the corresponding letter in $\operatorname{sort}(v(1), \ldots, v(i))$.

Let $\alpha=\left(\alpha_{1}, \alpha_{2}, \ldots\right)$ be a partition of $n$. It will be convenient to fix once and for all a numbering of the boxes of $\alpha$ : we number them by rows, from left to right, starting with the top row.

Notation: Let $T$ be a filling of $\alpha$ by the numbers 1 to $n$, with each number appearing once. Define $\pi(T)$ to be the permutation obtained by reading $T$ by rows from left to right, starting from the top row, that is $\pi(T)(i)$ is the number contained in box $i$.

We will be particularly interested in applying $\pi$ to SYTs, but we define it more generally for convenience.

Example: Let $\alpha=(2,2,1)$. Consider the following SYTs of shape $\alpha$ :

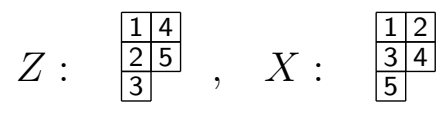

Note that $\pi(Z)=(1,4,2,5,3)$ and $\pi(X)=(1,2,3,4,5)$.

Proposition 4.4. Let $X, Z$ be standard Young tableaux of shape $\alpha$. The relation $Z \leqslant \mathrm{dom}$ $X$ holds if and only if $\pi(X) \leqslant \pi(Z)$ in the Bruhat order.

Proof. Let the sequence of partitions corresponding to $X$ be $\gamma^{(1)}, \ldots, \gamma^{(|\alpha|)}$, and let the sequence of partitions corresponding to $Z$ be $\delta^{(1)}, \ldots, \delta^{(|\alpha|)}$. Since $\pi(X) \leqslant \pi(Z)$ if and only if $\pi(X)^{-1} \leqslant \pi(Z)^{-1}$, we can apply the criterion of Theorem 4.3 to $\pi(X)^{-1}$ and $\pi(Z)^{-1}$. The sequence $\operatorname{sort}\left(\pi(X)^{-1}(1), \ldots, \pi(X)^{-1}(i)\right)$ consists of a sorted list of the positions of $1, \ldots, i$ in $X$, using the numbering of the boxes of $\alpha$ defined previously. The condition that $\operatorname{sort}\left(\pi(X)^{-1}(1), \ldots, \pi(X)^{-1}(i)\right)$ have each letter less than or equal to the corresponding letter in sort $\left(\pi(Z)^{-1}(1), \ldots, \pi(Z)^{-1}(i)\right)$ is equivalent to the condition that $\gamma^{(i)} \geqslant \operatorname{dom} \delta^{(i)}$; all these conditions put together yield exactly the condition that $X \geqslant_{\text {dom }} Z$.

Now we consider the "box move". We recall that a decreasing box move on $X$ swaps two entries of $X$, so that the larger entry moves higher, and the smaller entry moves lower. We have already established, in Lemma 2.1, that applying a decreasing box move moves us down in dominance order. We wish to prove the converse result, that if $X>Z$ in dominance order, then there is a sequence of decreasing box moves which takes us from $X$ to $Z$. Our strategy is inductive: we find a decreasing box move from $X$ to $Y$, such that $Y \geqslant Z$ in dominance order. In order to do this, we recast the problem in terms of Bruhat order on permutations.

Lemma 4.5. If $\pi(Y)$ covers $\pi(X)$ in Bruhat order, then there is a decreasing box move from $X$ to $Y$. 
Proof. A cover in Bruhat order swaps two entries, and moves the larger number up.

Therefore, our desired result follows once we prove the following result on permutations. Let us write $L \subseteq S_{n}$ for the set of permutations of the form $\pi(X)$ for $X$ a SYT of shape $\alpha$.

Proposition 4.6. If $x<z$ in Bruhat order, with $x, z \in L$, then there is a cover $x \lessdot y$ with $y \in L$ such that $y \leqslant z$.

Proof. Write a reduced expression for $z$ as a product of adjacent transpositions $s_{i_{1}} \cdots s_{i_{p}}$. Since $x<z$, by Theorem 4.2, there is a subword of this word which equals $x$. As in the proof of [1, Lemma 2.2.1], choose one such that the rightmost omitted transposition is as far to the left as possible (i.e. $x=s_{i_{1}} \cdots \widehat{s}_{i_{j_{1}}} \cdots \widehat{s}_{i_{j_{q}}} \cdots s_{i_{p}}$ with $j_{1}<\cdots<j_{q}$ such that $j_{q}$ is minimal, and $\widehat{s}$ means that $s$ is omitted). Define $y$ to be obtained from this subword by adding back in the rightmost transposition in the word for $z$ not in the chosen subword for $x$ (i.e., $y=s_{i_{1}} \cdots \widehat{s}_{i_{j_{1}}} \cdots \widehat{s}_{i_{j_{q-1}}} \cdots s_{i_{j_{q}}} \cdots s_{i_{p}}$ ). By the proof of [1, Lemma 2.2.1], this is a reduced expression for a permutation which covers $x$ in Bruhat order and lies below $z$.

We will now show that $y \in L$. We can write $y=x t$, where $t$ is the transposition $s_{i_{p}} s_{i_{p-1}} \ldots s_{i_{j_{q}}} \ldots s_{i_{p-1}} s_{i_{p}}$ (i.e., the product of the simple transpositions starting at the righthand end of our expression for $z$, proceeding backwards as far as $s_{i_{j_{q}}}$, and then proceeding forwards again to the end). From this description of $t$, it is clear that $\ell(z t)<$ $\ell(z)$, so $z t<z$ in Bruhat order.

Let $X$ and $Z$ denote the SYTs corresponding to $x$ and $z$ respectively. Let the transposition $t$ which we determined earlier be $(a, b)$ with $a<b$. Multiplying on the right by $t$ swaps the entries in positions $a$ and $b$. Since the effect of this on $z$ moves us down in Bruhat order, the larger entry must be in position $a$, and the smaller in position $b$. This implies that the boxes numbered $a$ and $b$ must be in distinct rows of the diagram for $\alpha$, since the entries of $Z$ within any single row are increasing. The same argument implies that $a$ and $b$ lie in distinct columns.

Now consider the effect of swapping the entries in positions $a$ and $b$ on $x$. We know that this yields $y=x t$ which covers $x$ in Bruhat order. Thus, the entry of $x$ in position $a$ is less than that in position $b$. Further, by Lemma 4.1, there are no entries with values between $x(a)$ and $x(b)$ and located between positions $a$ and $b$. This implies that swapping the entries in positions $a$ and $b$ of $X$ will result in a standard tableau, so $y \in L$, and we are done.

Remark: This proof for the implication $X>_{\text {dom }} Z \Longrightarrow X>_{\text {box }} Z$ is constructive as it exhibits the first box move: Let $s_{i_{1}} \cdots s_{i_{p}}$ be a reduced expression for $\pi(Z)$. Write $\pi(X)$ as a subword $s_{i_{1}} \cdots \hat{s}_{i_{j_{1}}} \cdots \hat{s}_{i_{j_{q}}} \cdots s_{i_{p}}$ such that $j_{q}$ is minimal. Then $s_{i_{p}} \cdots s_{i_{j_{q}}} \cdots s_{i_{p}}=(a, b)$ is a transposition and $\pi(X)(a, b)=\pi(Y)$ defines a standard Young tableau $Y$ which satisfies $Z \leqslant$ dom $Y$ and $Y<_{\text {box }} X$.

Example: Consider:

$$
Z: \begin{array}{|l|l|l|l|l|l|}
\hline 1 & 4 & 2 \\
\hline 2 & 5 \\
\hline 3
\end{array}, \quad X: \begin{array}{ll}
3 & 4 \\
\hline 5
\end{array}
$$


We have $\pi(Z)=14253$, and $\pi(X)=12345$. Let's say we want to move down from $X$. We write $\pi(Z)=(34)(23)(45)=s_{3} s_{2} s_{4}$. Now $\pi(X)=e$, so the subword of $s_{3} s_{2} s_{4}$ corresponding to $e$ is the empty subword. We add the rightmost reflection back in, so that is $(a, b)=s_{4}$. This gives us the permutation $y_{1}=12354$. To get the next step down the chain, we go to the subword of $z$ given by $y_{1}=s_{4}=12354$. Here we right multiply $y_{1}$ by $s_{4} s_{2} s_{4}=s_{2}$, obtaining 13254. In the final step we right multiply $y_{2}$ by $s_{4} s_{2} s_{3} s_{2} s_{4}=(2,5)$, obtaining $\pi(Z)$.

The corresponding sequence of fillings is:

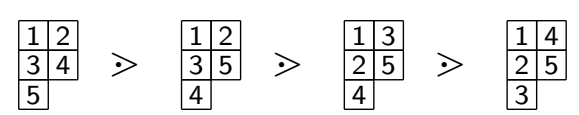

\section{The second proof of the main result}

Let $X, Z$ be SYTs of the same shape. In order to show that the dominance relation implies the box relation we present here a simple and explicit procedure to determine box moves on SYTs which transform $X$ into $Z$. If $Z<_{\text {dom }} X$, the following steps yield an SYT $Y$ such that $Z \leqslant$ dom $Y$ and $Y<_{\text {box }} X$.

(1) Find the largest entry $k$ which is in different positions in $X$ and $Z$.

(2) Let $a$ be the row of the entry $k$ in $Z$;

(3) let $m$ be the entry in that position in $X$, note that $m<k$;

(4) let $b>a$ be the first row below $a$ which contains an entry in the range $m<\cdot \leqslant k$ in $X$;

(5) let $\ell$ be the smallest entry with $m<\ell \leqslant k$ in row $b$ of $X$.

(6) The SYT $Y$ is obtained from $X$ by swapping the entries $\ell$ and $m$.

Example: The following SYTs $X$ and $Z$ have shape $(3,2,1)$ and are in dominance order:

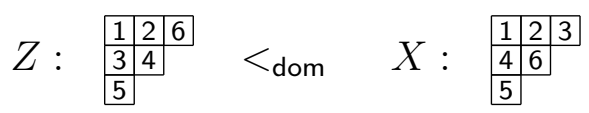

In the algorithm we compute: $k=6, a=1, m=3, b=2$ and $\ell=4$. Then

$$
Y: \begin{array}{ll|l|}
\hline 1 & 2 & 4 \\
\hline 3 & 6 & \\
\hline 5 & &
\end{array}
$$

satisfies $Z<_{\text {dom }} Y$ and we can continue. 
We illustrate this algorithm using tables which describe the difference of the two SYTs. Let $X$ and $Z$ be given by partition sequences $\left[\gamma^{(1)}, \ldots, \gamma^{(|\alpha|)}\right]$ and $\left[\delta^{(1)}, \ldots, \delta^{(|\alpha|)}\right]$, respectively. Each column of the table $T_{X-Z}$ is indexed by the partition number $j$, each row by the row number $i$. The entries are simply $\left(T_{X-Z}\right)_{i, j}=\gamma_{i}^{(j)}-\delta_{i}^{(j)}$.

In the above example, $X$ and $Z$ have partition sequences

$$
\begin{aligned}
& X \quad: \quad[(1),(2),(3),(3,1),(3,1,1),(3,2,1)] \text {, } \\
& Z \text { : }[(1),(2),(2,1),(2,2),(2,2,1),(3,2,1)] \text {, }
\end{aligned}
$$

respectively. Here is the corresponding table.

\begin{tabular}{c|c:c:c:c:c:c}
$T_{X-Z}$ & 1 & 2 & 3 & 4 & 5 & 6 \\
\hline 1 & 0 & 0 & 1 & 1 & 1 & 0 \\
\hdashline 2 & 0 & 0 & -1 & -1 & -1 & 0 \\
3 & 0 & 0 & 0 & 0 & 0 & 0
\end{tabular}

The table $T_{Y-Z}$ for the tableaux after the box move differs from $T_{X-Z}$ in column 3 which is zero.

The following lemma is obtained as a consequence of the definition of the dominance order.

Lemma 5.1. Two SYTs $X$ and $Z$ are in dominance order, $Z \leqslant_{\operatorname{dom}} X$, if and only if for each column $j$ and each row $a$ in the table $T_{X-Z}$, the sum $\sum_{i=1}^{a}\left(T_{X-Z}\right)_{i, j}$ of all entries in the $j$-th column from the top down to row a is nonnegative.

We can now give the second proof of Theorem 1.1.

Proof. For two SYTs $X, Z$ of the same shape $\alpha$ we need to show that they are in dominance order if and only if they are in box order. The "only if" part has been shown in Lemma 2.1. For the converse, assume that $X$ and $Z$ are in dominance order, say $Z \leqslant$ dom $X$. If $X$ and $Z$ are equal in dominace order, then $X=Z$ and we are done.

We show that in case $Z<_{\text {dom }} X$, the steps described above will produce a SYT $Y$ such that $Z \leqslant$ dom $Y$ and $Y<_{\text {box }} X$.

Consider the table $T_{X-Z}$, we discuss the entries as steps (1) through (4) are being

\begin{tabular}{|c|c|c|c|c|}
\hline$T_{X-Z}$ & $\ldots: m$ & $\cdots$ & $\ell-1$ & $\ell$ \\
\hline$\vdots$ & & & & \\
\hline$a$ & $>$ & $>$ & $\cdots$ & $\cdots$ \\
\hline$\vdots$ & $\geqslant$ & $\geqslant$ & & \\
\hline$\dddot{b}$ & & & & \\
\hline : & & & & \\
\hline$a^{\prime}$ & & & & \\
\hline$\vdots$ & & & & \\
\hline
\end{tabular}
performed.

(1) Let $k$ be the largest entry which is in different positions in $X$ and $Z$. 
By the choice of $k$, the partitions $\gamma^{(i)}, \delta^{(i)}$ are equal for $i \geqslant k$. Hence in the table, the $k$-th column, and everything on its right, is zero.

(2) Let $a$ be the row in which $k$ occurs in $Z$.

The entry $k$ occurs in different rows in $X$ and $Z$, say it occurs in row $a^{\prime}$ in $X$. Recall that $\delta^{(k)}=\gamma^{(k)}$, hence $\delta^{(k-1)}$ and $\gamma^{(k-1)}$ differ only in the two rows $a$ and $a^{\prime}$ where entry $k$ occurs in $Z$ and $X$, respectively. Then $\delta_{i}^{(k-1)}=\gamma_{i}^{(k-1)}$ for $i \neq a, a^{\prime}$ and

$$
\delta_{a}^{(k-1)}=\gamma_{a}^{(k-1)}-1, \quad \delta_{a^{\prime}}^{(k-1)}=\gamma_{a^{\prime}}^{(k-1)}+1 .
$$

Since $Z<_{\text {dom }} X$ are in dominance relation, we have $\delta^{(k-1)}<_{\text {dom }} \gamma^{(k-1)}$, hence $a^{\prime}>a$.

(3) Let $m$ be the entry in $X$ which has the position of $k$ in $Z$.

Note that $m<k$ since all entries greater than $k$ are in the same position in $X$ and $Z$. Considering the $a$-th rows of the two tableaux, it follows that

$$
\gamma_{a}^{(i)}>\delta_{a}^{(i)} \text { for } \quad m \leqslant i<k .
$$

(4) Let $b>a$ be the first row below $a$ which contains an entry in the range $m<\cdot \leqslant k$ in $X$.

Such a row $b$ exists since $a^{\prime}>a$ and row $a^{\prime}$ contains the entry $k$. Consider a row $r$ in the table $T_{X-Z}$ with $a<r<b$. The entries greater than $k$ are in the same positions in $X$ and in $Z$, and none of the entries in the range $m<\cdot \leqslant k$ occur in row $r$ in $X$, hence for each entry $s$ with $m<s<k, \gamma_{r}^{(s)}-\delta_{r}^{(s)} \geqslant 0$. Thus, the entries in $T_{X-Z}$ in the rectangle given by rows $m \leqslant \cdot<k-1$ and columns $a<\cdot<b$ are all nonnegative. This finishes the proof that the given entries in the table $T_{X-Z}$ are as specified.

(5) Let $\ell$ be the minimal entry in row $b$ of $X$ with $m<\ell \leqslant k$.

We claim that the column in $X$ containing $\ell$ is on the left of the column in $X$ containing $m$. For this, recall that $Z$ has $k$ in the position where $X$ has $m$. In the quadrant underneath and to the right of $k$ in the SYT $Z$, all entries are greater than or equal to $k$. Such entries are in the same position in $X$ and $Z$, which implies the claim.

(6) To obtain $Y$ from $X$, exchange the entries $\ell$ and $m$.

We show that $Y$ is a SYT. By the choice of $\ell$, the entries in row $b$ of $Y$ are strictly increasing; by the choice of $k$ and $m$, the entries in row $a$ of $Y$ are strictly increasing.

Now, consider the column of $Y$ that contains the entry $m$. Since $m<\ell$ and $X$ is a SYT, all entries in rows of higher numbers are bigger than $m$. Assume that there exists an entry $n>m$ in the column of $m$ and above $m$. By the choice of $b$ (minimality), it follows that $n$ is in row $a$ or above. This is a contradiction, because $X$ is a SYT.

Regarding the column of $Y$ that contains the entry $\ell$, recall that this entry is in the position where $X$ has $m$ and $Z$ has $k$. Since both $X$ and $Z$ are SYTs, the entries in this column in $Y$ must be strictly increasing. This finishes the proof that $Y$ is a SYT, and, using the claim under (5), that $Y$ is obtained from $X$ by a decreasing box move.

It remains to prove that $Z \leqslant \operatorname{dom} Y$. For this, we first verify that the table $T_{X-Y}$ for the tableaux $X$ and $Y$ has only zero entries, except in the positions indicated. This is 
straightforward since $Y$ is obtained from $X$ by a box move which exchanges entries $\ell$ and $m$ in rows $a$ and $b$.

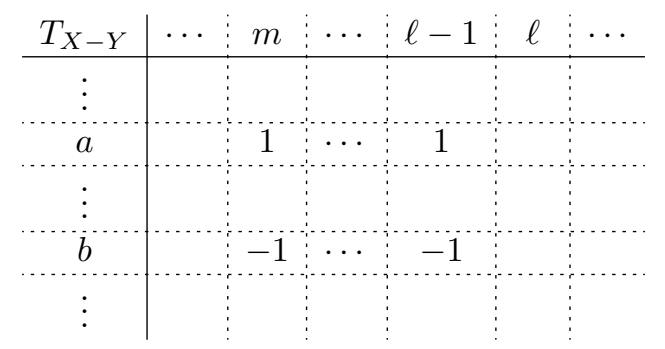

The table $T_{Y-Z}$ is obtained by subtracting the entries in $T_{X-Y}$ from those in $T_{X-Z}$. Since $X$ is a SYT, the columns for $T_{X-Z}$ have nonnegative partial sums, as specified in Lemma 5.1. Using the particular form of $T_{X-Z}$ established in steps (1) through (4), it follows that also the columns for $T_{Y-Z}$ have nonnegative partial sums. Applying Lemma 5.1 again yields $Y \geqslant$ dom $Z$.

We have seen that $Y$ is obtained from $X$ by a decreasing box move. By repeating this process, starting with the SYTs $Y$ and $Z$, we produce in finitely many rounds the desired sequence of box moves.

\section{Combinatorial properties of the order $\leqslant_{\text {box }}$}

In this section we study combinatorial properties of the poset $\left(\mathcal{T}_{\alpha}, \leqslant_{\text {box }}\right)$, and discuss in Section 6.2 an application to invariant subspaces of nilpotent linear operators.

\subsection{An example and some properties of $\leqslant$ box}

Here is the poset $\left(\mathcal{T}_{(3,2,1)}, \leqslant\right.$ box $)$.

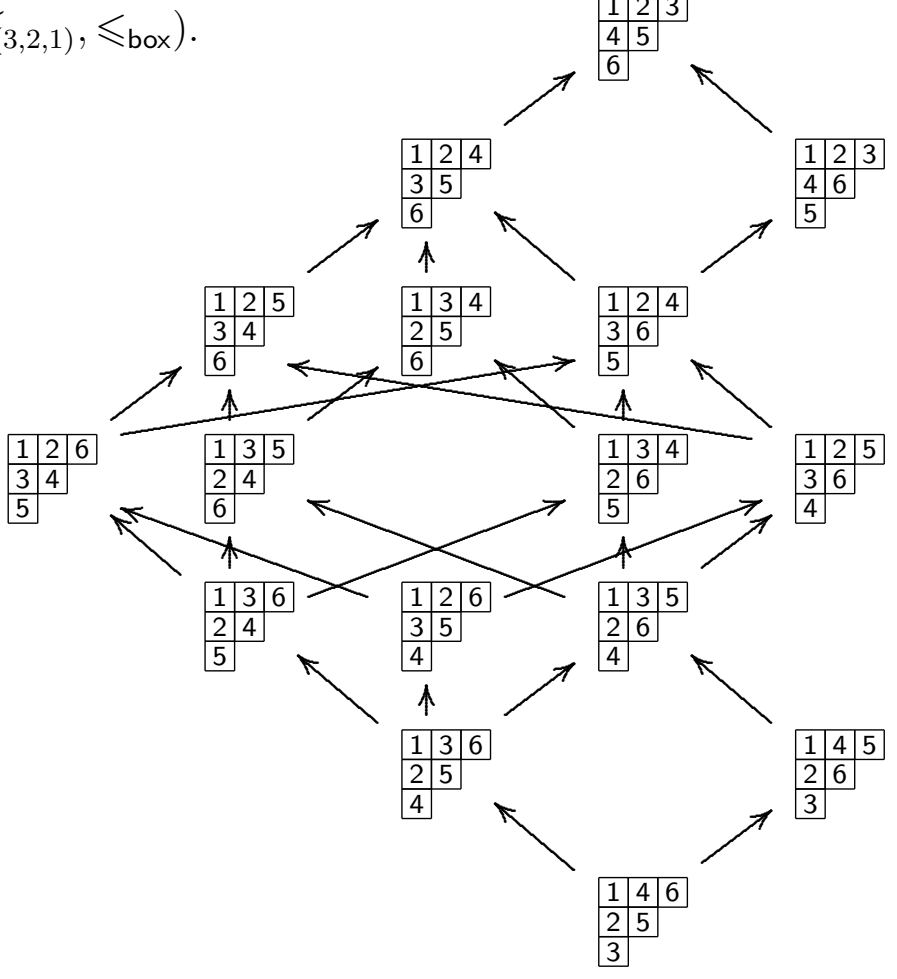


We observe:

- in this poset there exists exactly one maximal and exactly one minimal element;

- all saturated chains have the same length;

- this poset is not a lattice.

The first two properties hold for each poset of the form $\left(\mathcal{T}_{\alpha}, \leqslant\right.$ box $)$. First, we verify that the poset has a unique maximal and a unique minimal element.

Lemma 6.1. Let $\alpha$ be a partition.

1. There is a unique maximal element $X$ in the poset $\left(\mathcal{T}_{\alpha}, \leqslant_{\text {box }}\right)$. The $S Y T$ for $X$ is given by writing the numbers $1, \ldots,|\alpha|$ in numerical order into the Young diagram for $\alpha$.

2. There is a unique minimal element $Z$ in the poset $\left(\mathcal{T}_{\alpha}, \leqslant_{\text {box }}\right)$. The $S Y T$ for $Z$ is the transpose of the Young diagram for $\alpha^{\prime}$ in which the numbers $1, \ldots,|\alpha|$ are entered in numerical order.

Proof. 1. If two numbers $i, i+1$ do not occur in numerical order in the SYT for $X$, then the box move exchanging $i$ with $i+1$ yields a SYT which is larger in the dominance order.

2. By definition, taking transposes is an order-reversing bijection between the posets $\left(\mathcal{T}_{\alpha}, \leqslant_{\text {box }}\right)$ and $\left(\mathcal{T}_{\alpha^{\prime}}, \leqslant_{\text {box }}\right)$.

Next, we verify that all saturated chains in these posets have the same length.

Proposition 6.2. For a partition $\alpha$, all saturated chains in the poset $\left(\mathcal{T}_{\alpha}, \leqslant_{\text {box }}\right)$ have the same length.

Proof. Since covers in the Bruhat order increase the length by 1 (Lemma 4.1), the result follows from Theorem 1.1, Proposition 4.4, Lemma 4.5 and Proposition 4.6.

\subsection{An application to invariant subspaces}

We return to the link between LR-tableaux and varieties of invariant subspaces of nilpotent linear operators studied in Section 3.

It follows from Lemma 6.1 that the poset $\left(\mathcal{T}_{\alpha, \gamma}^{\beta}, \leqslant\right.$ dom $)$ has a unique minimal and a unique maximal element provided the partitions $\alpha, \beta, \gamma$ are such that $\beta \backslash \gamma$ is a rook strip. This extends the corresponding result [5, Proposition 5.5] for the case where $\beta$ and $\gamma$ are arbitrary but all parts of $\alpha$ are at most 2 .

In terms of varieties of invariant subspaces, Lemma 6.1 has the following interpretation. 
Corollary 6.3. Given partitions $\alpha, \beta, \gamma$ such that $\beta \backslash \gamma$ is a rook strip, the variety

$$
\mathbb{V}_{\alpha, \gamma}^{\beta}=\bigcup_{X \in \mathcal{T}_{\alpha, \gamma}^{\beta}} \mathbb{V}_{X}
$$

has a unique open component $\mathbb{V}_{O}$ and a unique closed component $\mathbb{V}_{C}$. The open component $\mathbb{V}_{O}$ and the closed component $\mathbb{V}_{C}$ are given by the LR-tableaux $O$ and $C$, respectively, which correspond to the unique minimal and the unique maximal element in the poset $\left(\mathcal{T}_{\alpha, \gamma}^{\beta}, \leqslant\right.$ dom $)$.

Proof. The following statements are equivalent for an LR-tableau $O \in \mathcal{T}_{\alpha, \gamma}^{\beta}$ : (1) $O$ is a minimal element in the poset $\left(\mathcal{T}_{\alpha, \gamma}^{\beta}, \leqslant_{\text {dom }}\right) ;(2) O$ is a minimal element in the poset $\left(\mathcal{T}_{\alpha, \gamma}^{\beta}, \leqslant\right.$ boundary $) ;(3)$ there is no $X \in \mathcal{T}_{\alpha, \gamma}^{\beta} \backslash\{O\}$ such that $\mathbb{V}_{O} \cap \overline{\mathbb{V}}_{X} \neq \varnothing ;$ (4) the union $\cup_{X \neq O} \mathbb{V}_{X}$ is closed in $\mathbb{V}_{\alpha, \gamma}^{\beta} ;(5)$ the variety $\mathbb{V}_{O}$ is open in $\mathbb{V}_{\alpha, \gamma}^{\beta}$. Hence, since the poset $\left(\mathcal{T}_{\alpha, \gamma}^{\beta}, \leqslant\right.$ dom $)$ has $O$ as the unique minimal element, the component $\mathbb{V}_{O}$ is the unique open component in the decomposition in the lemma.

Similarly, the following statements are equivalent for $C \in \mathcal{T}_{\alpha, \gamma}^{\beta}:(1) C$ is a maximal element in the poset $\left(\mathcal{T}_{\alpha, \gamma}^{\beta}, \leqslant\right.$ dom $) ;(2) C$ is a maximal element in the $\operatorname{poset}\left(\mathcal{T}_{\alpha, \gamma}^{\beta}, \leqslant_{\text {boundary }}\right)$; (3) there is no $X \in \mathcal{T}_{\alpha, \gamma}^{\beta} \backslash\{C\}$ such that $\mathbb{V}_{X} \cap \overline{\mathbb{V}}_{C} \neq \varnothing$; (4) the variety $\mathbb{V}_{C}$ is closed in $\mathbb{V}_{\alpha, \gamma}^{\beta}$. The uniqueness of the maximal element $C$ in $\left(\mathcal{T}_{\alpha, \gamma}^{\beta}, \leqslant\right.$ dom $)$ implies that $\mathbb{V}_{C}$ is the unique closed component in the decomposition.

We now consider some examples in which we relax the condition that $\beta \backslash \gamma$ is a rook strip. We note that in this case, the definition of box moves is modified to allow resorting the rows (if $\beta \backslash \gamma$ is a horizontal strip) or the columns (if $\beta \backslash \gamma$ is a vertical strip).

Example: $\quad 1$. The first example shows that the condition that $\beta \backslash \gamma$ be a horizontal strip is needed for the uniqueness of the maximal element in $\mathcal{T}_{\alpha, \gamma}^{\beta}$. Consider the partition triple $\beta=(4,3,2,2,1), \gamma=(3,2,1,1)$ and $\alpha=(2,2,1)$. The Hasse diagram of the poset $\left(\mathcal{T}_{\alpha, \gamma}^{\beta}, \leqslant_{\text {dom }}\right)$ (or equivalently of $\left(\mathcal{T}_{\alpha, \gamma}^{\beta}, \leqslant\right.$ box $)$ ) has the following shape:

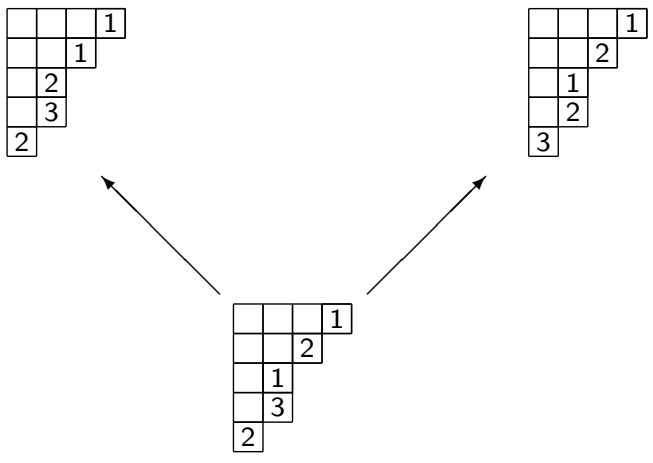

2. The second example shows that in Theorem 1.1, the condition that $\beta \backslash \gamma$ be a vertical strip is necessary. We also see that for horizontal strips, the poset $\left(\mathcal{T}_{\alpha, \gamma}^{\beta}, \leqslant\right.$ box $)$ may have several minimal and several maximal elements.

Let $\beta=(5,4,3,1), \gamma=(4,3,2,1)$ and $\alpha=(2,2,1)$. There are two LR-fillings of type $(\alpha, \beta, \gamma)$ : 

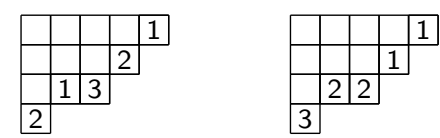

They are incomparable in $\leqslant_{\text {box }}$ relation, but

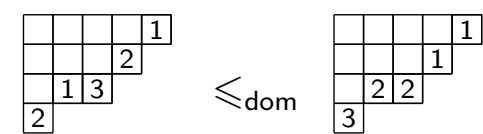

\subsection{Open Question}

We want to compare the two algorithms presented above. Suppose we have two SYTs $X, Z$ of shape $\alpha$, such that $X>_{\text {dom }} Z$. Say the first algorithm produces a SYT $\tilde{Y}$, the second a SYT $Y$, both of shape $\alpha$. The tableaux satisfy

$$
X>_{\text {dom }} \tilde{Y} \geqslant_{\text {dom }} Z, \quad X>_{\text {dom }} Y \geqslant_{\text {dom }} Z .
$$

The first algorithm depends on the choice of a factorization of $z$ as a product of adjacent transpositions. In this section, we describe a way to make the choice so that (conjecturally) the two algorithms produce the same result, i.e., so that $Y=\tilde{Y}$. Suppose that to produce the reduced expression for $z \in S_{n}$, a version of the "bubble sort" algorithm is used, as in [1, Example 3.4.3].

Starting from the permutation $z$, we will find a sequence of adjacent transpositions which transform $z$ into the identity permutation. Start by moving $n$ into the final position. If $n$ started in position $u$, this gives us the permutation $z \cdot s_{u} \cdots s_{n-1}=z \cdot z_{(n)}^{-1}$. Next, move entry $n-1$ into its proper position, which gives us $z \cdot z_{(n)}^{-1} \cdot s_{u^{\prime}} \cdots s_{n-2}=z \cdot z_{(n)}^{-1} \cdot z_{(n-1)}^{-1}$, where $u^{\prime}$ was the position of $n-1$ in $z \cdot z_{(n)}^{-1}$. Continue with the entry $n-2$ and so forth. We obtain $e=z \cdot z_{(n)}^{-1} \cdots z_{(2)}^{-1}$. In other words, $z_{(2)} \cdots z_{(n)}$ is a reduced expression for $z$.

Conjecture 6.4. If we apply the first algorithm with the choice of reduced expression described above, its result agrees with the result of applying the second algorithm.

\section{Acknowledgements}

The authors would like to thank the referees for very helpful comments which have led to substantial improvements of the paper.

\section{References}

[1] A. Björner and F. Brenti. Combinatorics of Coxeter groups. Graduate Texts in Mathematics 231. Springer Verlag, Berlin, 2005.

[2] W. Fulton. Young Tableaux. Cambridge University Press, 1997.

[3] T. Klein. The multiplication of Schur-functions and extensions of $p$-modules. $J$. Lond. Math. Soc., 43: 280-284, 1968. 
[4] J. Kosakowska and M. Schmidmeier. Operations on arc diagrams and degenerations for invariant subspaces of linear operators. Trans. Amer. Math. Soc., 367: 5475-5505, 2015.

[5] J. Kosakowska and M. Schmidmeier. Arc diagram varieties. Contemporary Mathematics series of the AMS, 607: 205-224, 2014.

[6] J. Kosakowska and M. Schmidmeier. The boundary of the irreducible components for invariant subspace varieties. Math. Z., 290: 953-972, 2018.

[7] I. G. Macdonald. Symmetric Functions and Hall Polynomials. Oxford University Press, 1995.

[8] M. Schmidmeier. The entries in the LR-tableau. Math. Z., 268: 211-222, 2011.

[9] M. Taşkin. Properties of four partial orders on standard Young tableaux. J. Combin. Theory Ser. A, 113: 1092-1119, 2006. 